\title{
A novel ZEB1/HAS2 positive feedback loop promotes EMT in breast cancer
}

\author{
Bogdan-Tiberius Preca ${ }^{1,2,3}$, Karolina Bajdak ${ }^{1}$, Kerstin Mock ${ }^{1}$, Waltraut Lehmann $^{1}$, \\ Vignesh Sundararajan ${ }^{1}$, Peter Bronsert ${ }^{2,4,5}$, Alexandra Matzge-Ogi ${ }^{6,7}$, Véronique \\ Orian-Rousseau ${ }^{6}$, Simone Brabletz ${ }^{8}$, Thomas Brabletz ${ }^{8}$, Jochen Maurer ${ }^{1,2,3, *}$, Marc \\ P. Stemmler ${ }^{8 * *}$ \\ ${ }^{1}$ Department of General and Visceral Surgery, Medical Center, University of Freiburg, Faculty of Medicine, Freiburg, Germany \\ ${ }^{2}$ German Cancer Consortium (DKTK), Heidelberg, Germany \\ ${ }^{3}$ German Cancer Research Center (DKFZ), Heidelberg, Germany \\ ${ }^{4}$ Institute for Surgical Pathology, Medical Center - University of Freiburg, Faculty of Medicine, Freiburg, Germany \\ ${ }^{5}$ Tumorbank Comprehensive Cancer Center Freiburg, Medical Center, University of Freiburg, Faculty of Medicine, Freiburg, \\ Germany \\ ${ }^{6}$ Karlsruhe Institute of Technology, Institute of Toxicology and Genetics, Eggenstein-Leopoldshafen, Germany \\ ${ }^{7}$ Amcure GmbH, Eggenstein-Leopoldshafen, Germany \\ ${ }^{8}$ Department of Experimental Medicine I, Nikolaus-Fiebiger Center for Molecular Medicine, Friedrich-Alexander University of \\ Erlangen-Nürnberg, Erlangen, Germany \\ *These authors contributed equally to this work
}

Correspondence to: Marc P. Stemmler, email: marc.stemmler@fau.de

Keywords: hyaluronic acid synthase 2 (HAS2), epithelial-mesenchymal transition, invasion, metastasis, CD44 signaling

Received: August 15, 2016

Accepted: December 26, 2016

Published: January 09, 2017

\section{ABSTRACT}

Cancer metastasis is the main reason for poor patient survival. Tumor cells delaminate from the primary tumor by induction of epithelial-mesenchymal transition (EMT). EMT is mediated by key transcription factors, including ZEB1, activated by tumor cell interactions with stromal cells and the extracellular matrix (ECM). ZEB1mediated EMT and motility is accompanied by substantial cell reprogramming and the acquisition of a stemness phenotype. However, understanding of the underlying mechanism is still incomplete. We identified hyaluronic acid (HA), one major ECM proteoglycan and enriched in mammary tumors, to support EMT and enhance ZEB1 expression in cooperation with CD44s. In breast cancer cell lines HA is synthesized mainly by HAS2, which was already shown to be implicated in cancer progression. ZEB1 and HAS2 expression strongly correlates in various cancer entities and high HAS2 levels associate with an early relapse. We identified HAS2, tumor cell-derived HA and ZEB1 to form a positive feedback loop as ZEB1, elevated by HA, directly activates HAS2 expression. In an in vitro differentiation model HA-conditioned medium of breast cancer cells is enhancing osteoclast formation, an indicator of tumor cell-induced osteolysis that facilitates formation of bone metastasis. In combination with the previously identified ZEB1/ESRP1/CD44s feedback loop, we found a novel autocrine mechanism how ZEB1 is accelerating EMT.

\section{INTRODUCTION}

Breast cancer is the most frequently diagnosed cancer in women with over 1.7 Million new cases and more than 500,000 patients succumbing to the disease every year. While the primary tumor is often detected and removed by surgery, $20-40 \%$ of patients suffer from tumor relapse due to cancer-cell spreading. These metastases that are frequently found in bones, lung, liver and the brain are the major cause for cancer-related deaths today $[1,2]$. One critical event during cancer progression is the acquisition of a plastic, mesenchymal and motile phenotype by tumor 
cells originating from epithelial tissue. This allows tumor cells to delaminate from the primary tumor, break through the basement membrane, invade the surrounding tissues and eventually enter the blood stream. Upon transport to distant sites they extravasate from blood vessels and form micrometastases [3, 4]. This metastatic cascade requires the activation of embryonic programs: during cell spreading the epithelial-mesenchymal transition (EMT) is activated, followed by the induction of the reverse process, the mesenchymal-epithelial transition (MET), to allow colonization. The resulting metastases often resemble the primary tumor in grading and marker gene expression $[1,4,5]$. Molecularly, EMT is induced by the action of specific transcription factors of the ZEB (ZEB1/2), Snail (SNAI1/2) and basic helix loop helix families (TWIST1) [6-8]. It was shown that ZEB1 is a major driver of EMT, tumorigenesis and metastasis formation. It provides stemness properties, resistance to chemotherapy and its expression correlates with poor prognosis [9-13]. ZEB1 acts mainly as transcriptional repressor, regulating genes involved in cell adhesion, cell polarity and tight junctions as well as key epithelial microRNAs. In particular the miR-200 family that induces and stabilizes epithelial differentiation is embedded in a double negative regulatory feedback loop with ZEB1 [12, 14-16]. Moreover, ZEB1 induces epigenetic changes and cooperates with the Hippo-transducer YAP1 to also act as transcriptional activator of specific target genes involved in stemness, invasion and metastasis $[9,17,18]$. We recently found that ZEB1 also regulates differential splicing of the stem cell marker CD44. By repressing ESRP1 epithelial-specific CD44v isoforms are switched to the standard isoform CD44s that further enhances ZEB1 expression to maintain an EMT phenotype even in absence of external EMT stimuli [19].

Although these findings in part explain the molecular downstream function of ZEB1 within the tumor cell, efficient invasion and metastasis require interaction with the extracellular matrix (ECM) and the surrounding stroma as well. It is well known that tumor cells influence ECM composition to facilitate migration and invasion into the surrounding tissues [20, 21]. Hylaruronan (hyaluronic acid, HA) is one ubiquitously expressed simple proteoglycan that is present in the ECM. It is required for proper embryogenesis and regeneration, but often becomes deregulated in disease [20]. HA forms scaffolds for ECM assembly, functions as hydrogel to complex water molecules and directly signals to cells by interacting with a variety of cell surface receptors, including CD44 [20, 22]. $\mathrm{HA}$ is synthesized in different chain lengths differing in molecular weight and molecular function [23]. It was demonstrated that HA molecular weight composition is altered during tumorigenesis and that this alteration plays a major role in tumor progression [24, 25]. The tumor and metastasis promoting function is mediated in part by HA binding to and subsequent activation of CD44 [26, 27].
Autocrine and paracrine signals instruct tumor and stroma cells to deposit HA into the ECM, synthesized by three hyaluronic acid synthases (HAS1-3) [28]. HAS2 was shown to play a crucial role in the context of tumorigenesis. Elevated HAS2 expression was correlated with an EMT phenotype in over $70 \%$ of metaplastic breast carcinoma [29]. Recently, it was shown that excess of HA generated by a $H A S 2$ transgene in a mouse model for breast cancer, accelerated the development of carcinoma [30].

Here we analyzed whether tumor cell secreted HA and $H A S 2$ expression is promoting ZEB1-dependent EMT and found that HA in combination with CD44s activates ZEB1 expression. ZEB1 promotes additional HA synthesis by activation of $H A S 2$, thereby generating an additional self-enforcing feedback loop involving HA/CD44s, ZEB1 and HAS2.

\section{RESULTS}

\section{Extracellular hyaluronic acid triggers $Z E B 1$ expression}

EMT and malignancy are ultimately connected with ECM reconstruction. Deposition of excess HA plays an important pro-invasive and pro-metastatic role [31]. We aimed to dissect how increased extracellular HA contributes to ZEB1-driven EMT and how its synthesis and secretion is regulated during tumor progression.

We made use of the triple-negative breast cancer cell line MDA-MB231 and its descendent line MDABoM1833, which has been selected for increased capacity to form bone metastasis upon injection of the parental cell line in mice [32]. Treatment of these two mesenchymal-like malignant cell lines with HA induced an increase in ZEB1 protein levels (Figure 1A). This 24-h short term treatment did not result in ZEB1-dependent CD44s accumulation yet. In contrast, addition of HA to the epithelial and noninvasive cancer cell line MCF7 and the mammary fibrocystic cell line MCF10A had rather opposite effects leading to further reduction of the already low levels of ZEB1, likely owing to the fact that one important receptor of HA, CD44s, is not expressed in MCF7 and MCF10A (Figure 1A) [19]. In line with this, overexpression of $C D 44 \mathrm{~s}$ and treatment with extracellular HA showed a very robust upregulation of ZEB1 in MCF7 cells (Figure 1B). Hence, HA supports ZEB1-driven EMT that is enhanced by CD44s.

\section{HAS2 in breast cancer cell lines is crucial for autocrine HA-dependent activation of $Z E B 1$}

To further investigate this finding we interfered with autocrine HA synthesis by 4-methylumbelliferone (4-MU) treatment [33, 34]. MDA-MB231 and MDABoM1833 cells showed increased levels of HA secretion in comparison to the epithelial cell lines MCF7 and 
MCF10A (Figure 1C). Blocking HA synthesis in MDA-MB231 and MDA-BoM1833 resulted in robust downregulation of ZEB1 that coincided with reduction in CD44s and upregulation of E-cadherin (E-cad) on protein and mRNA levels, indicating activation of EMT (Figure $1 \mathrm{D}$ and 1E; Supplementary Figure S1A). Interestingly, 4-MU treatment also induced downregulation of total CD44 levels in contrast to a ZEB1 knockdown that induced CD44 isoform switching as shown previously (Supplementary Figure S1A) [19].

HA is synthesized by three different hyaluronic acid synthases (HAS) encoded by HAS1-3, with different properties concerning the molecular size and function of the generated HA. In tumors HA is synthesized and secreted by stromal as well as by cancer cells. As $H A S 2$ expression and HAS2-generated HA have been shown to promote tumorigenesis, we asked whether HAS2 activity induces $Z E B 1$ expression in cancer cells. We used siRNAmediated gene silencing that resulted in efficient $H A S 2$ knockdown and reduced the amount of secreted HA to $50 \%$ and $10 \%$ in MDA-MB231 and MDA-BoM1833 cells, respectively (Figure 1F and 1G; Supplementary Figure S1B). This resulted in a slight downregulation of ZEB1 protein levels only in MDA-BoM1833 cells, whereas transcripts were reduced to $50 \%$ and $40 \%$ in MDA-MB231 and MDA-BoM1833 cells, respectively (Figure 1F and Supplementary Figure S1B). Similar to the treatment with 4-MU, knockdown of HAS2 led to a reduction of $C D 44 s$-specific transcripts below $60 \%$ in both cell lines. Interestingly, the effect of $H A S 2$ knockdown was not further enhanced by simultaneous depletion of all three HAS genes. Synthesis and secretion of HA was not blocked more efficiently, confirming that the majority of HA is produced by HAS2 whereas HAS1 and HAS3 play only minor roles in this context (Figure $1 \mathrm{G}$ and Supplementary Figure S1C). Taken together, these results show that secreted HA plays a key role in regulating ZEB1 in an autocrine manner. Specifically the enzymatic activity of HAS2 is producing HA and promotes ZEB1-induction.

\section{$H A S 2$ strongly correlates with $Z E B 1$ expression in tumors and poor prognosis}

We wanted to understand whether EMT marker expression and HAS2 were correlated in cell lines and tumor samples. MDA-MB231 and MDA-BoM1833 cells with a mesenchymal phenotype showed low expression of $E$-cad, whereas $Z E B 1$ and $C D 44 s$ were highly expressed (Figure 2A and 2B). In line with increased HA secretion, $H A S 2$ was detected at substantial levels in these cell lines by Western blotting, immunofluorescence labeling and qRT-PCR analysis (Figure 2A and 2C, Supplementary Figure S2A). In contrast, the epithelial breast cancer and fibrotic cell lines MCF7 and MCF10A showed high $E$-cad levels and low levels of ZEB1, CD44s and $H A S 2$, reflecting a weak or absent EMT signature (Figure $2 \mathrm{~A}-2 \mathrm{C}$,
Supplementary Figure S2A). HAS3 expression was indifferent in all cell lines and $H A S 1$ transcripts were not detectable (Figure 2C, data not shown). In breast cancer tissue sections we found robust co-expression of ZEB1 and HAS2 in tumor cell areas, whereas tumor cells missing ZEB1 were also negative for HAS2 (Figure 2D, Supplementary Figure S2B). This was also reflected by a correlation analysis of $Z E B 1$ and $H A S 2$ expression in microarray data sets of the CCLE (GSE3613332) [35] and the 'NCI60' (GSE58463) [36] panels (Figure 2E and Supplementary Figure S3). Moreover, genomewide transcript analysis of tumor samples confirmed a close correlation between $Z E B 1$ and $H A S 2$ (but not with $H A S 1$ and $H A S 3$ ) in breast, pancreas and lung cancer specimens (Figure 2F) (GSE42568 [37], GSE28735 [38] and GSE41271 [39]). Strikingly, high and low HAS2 expression levels were associated with differences in the period to relapse (Figure 2G and Supplementary Figure S4). High HAS2 levels alone already showed correlation with poor prognosis (Supplementary Figure S4C). Moreover, elevated levels of a HAS2, CD44 and ZEB1 geneset increased the hazard ratio from 2.84 to 11.47 in relapse-free and from 3.147 to 22.78 in overall survival studies (Figure 2G, Supplementary Figure S4A and S4C). Interestingly, the aggressive claudin-low subtype of triple-negative breast cancers showed high expression of $H A S 2, C D 44$ and ZEB1 (Figure 2H and Supplementary Figure S4D). HAS1 and $H A S 3$ showed no correlation to survival (Supplementary Figure S4B and S4C). These results indicate that $H A S 2, C D 44$ and $Z E B 1$ expression is correlated in cell lines and tumor samples. HAS2 is involved in tumor progression and acts in concert with ZEB1-driven EMT.

\section{HAS2 is necessary during TGF $\beta$-induced EMT}

To gain further insights into the dynamic changes of HAS2 and ZEB1 levels during EMT and how they control each other's expression after initiation of EMT, we utilized in vitro systems to induce EMT. MCF10A cells are fibrocystic mammary epithelial cells without tumorigenic potential in vivo, but they can undergo EMT upon TGF $\beta$ stimulation or by induction of exogenous $Z E B 1$ expression [19, 40]. Experimental induction of $Z E B 1$ from a stably transfected doxycycline (Dox)-inducible expression construct in MCF10A cells raised the levels of HAS2 protein after seven days of Dox treatment, but not in empty vector control cells (Figure 3A). Long-term TGF $\beta$ stimulation of wildtype MCF10A cells for 21 days had similar effects. During EMT induction, ZEB1 levels were increased, whereas E-cad became downregulated. Concomitantly, HAS2 expression and CD44s splicing were induced, as evident on protein level (Figure 3B). In line with increased HAS2 levels by TGF $\beta$ treatment, secreted HA was elevated (Figure 3C). We next analyzed MCF10A cells in shorter intervals during the 21 days 
of TGF $\beta$ treatment to observe dynamic changes in gene expression during EMT by qRT-PCR. In agreement with the endpoint analysis and our previous observations, we found a gradual increase of $C D 44 s, Z E B 1$, vimentin and a reduction of $E$-cad transcripts, whereas total $C D 44$ levels remained constant, presumably due to alterations in ESRP1-regulated splicing (Figure 3D) [19, 41]. $H A S 2$ showed a similar gradual increase of transcript levels over the entire duration of TGF $\beta$ treatment and the fold-changes between $Z E B 1$ and $H A S 2$ were of the same range, suggesting that their expression is linked and regulated by a common mechanism (Figure 3D). Knockdown of HAS2 in MCF10A cells during the 21 days of TGF $\beta$ treatment resulted in a complete block of EMT. ZEB1 levels were not increased, CD44 differential splicing was not shifted towards $C D 44 s$ and $E$-cad was not downregulated. In comparison to untreated cells, siRNA mediated HAS2 knockdown inverted the effects of TGF $\beta$ and even pushed the cells towards a more epithelial phenotype with reduction in ZEB1, vimentin and CD44s and increased $E$-cad levels at the end of the treatment (Figure 3E). Knockdown of HAS2 during TGF $\beta$ treatment
A

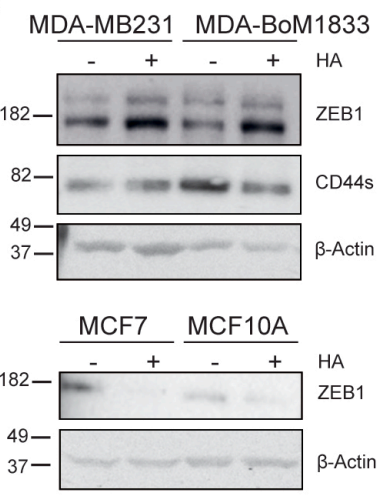

B

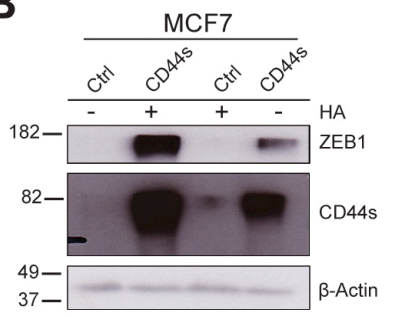

C

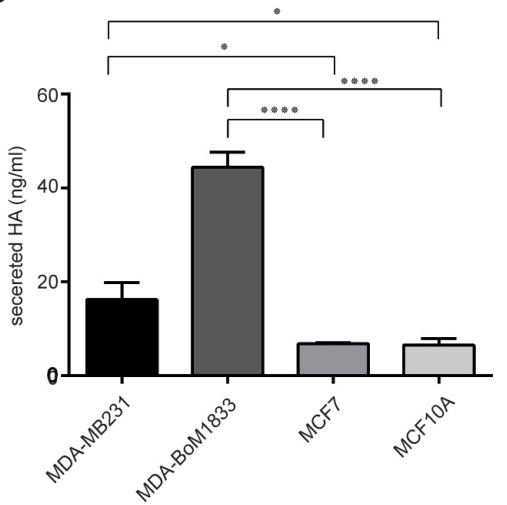

D

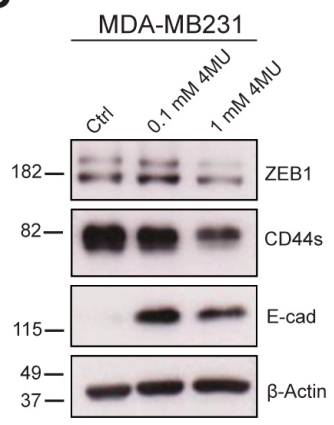

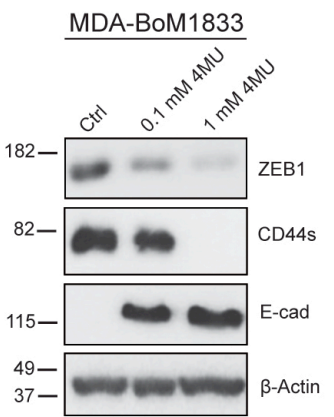

$\mathbf{F}$

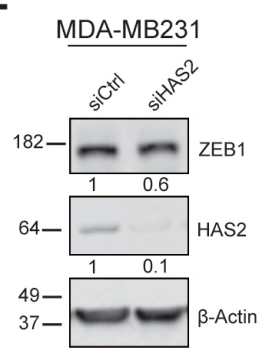

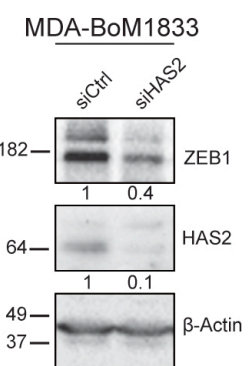

E
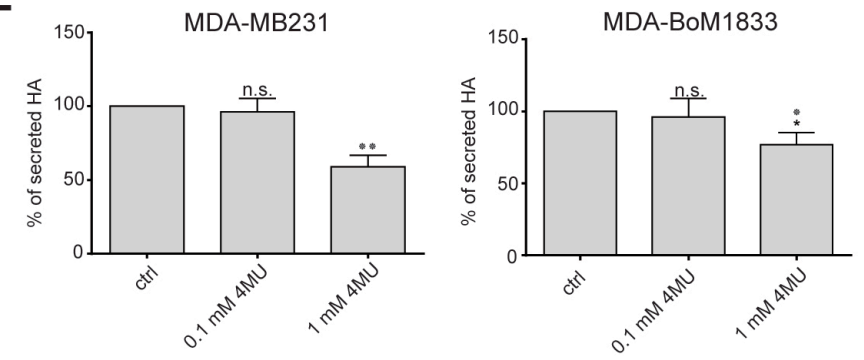

G
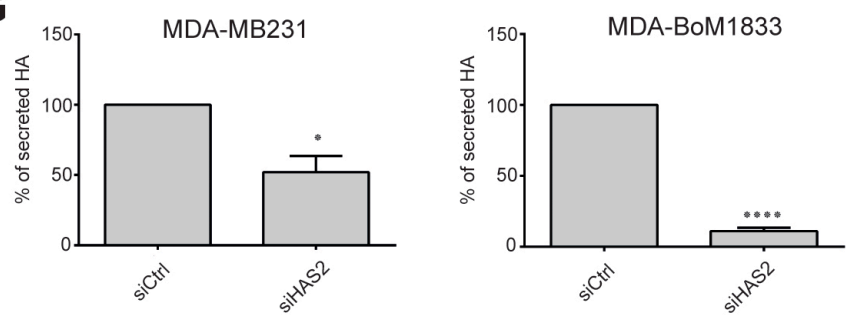

Figure 1: Hyaluronic acid (HA) is activating ZEB1 and CD44s expression. (A) Western blot of mesenchymal and epithelial breast cancer cell lines showing increasing ZEB1 levels upon HA treatment in MDA-MB231 and MDA-BoM1833 whereas welldifferentiated MCF7 and non-tumorigenic MCF10A cells are not affected. (B) ZEB1 protein levels in Western blots are increased upon combined CD44s transfection and HA treatment of MCF7 cells. Exogenous CD44s is stabilized and increased by HA treatment. (C) Measurement of secreted HA levels in breast cancer cell lines reveal increased levels in cells with a mesenchymal phenotype. (D) Blocking of HA production by 4-MU is inducing MET in MDA-MB231 and MDA-BoM1833 cells evident by decreasing ZEB1 and CD44s levels and activation of E-cad in Western blot (E) Quantification of secreted HA upon 4-MU treatment. (F) Western blot of MDA-MB231 and MDA-BoM1833 cells upon knockdown of HAS2 shows ZEB1 downregulation, verified by quantification as indicated by numbers below individual blots. (G) Quantification of secreted HA in MDA-MB231 and MDA-BoM1833 upon HAS2 knockdown reveals that HAS2 is the main enzyme for HA synthesis. 
A

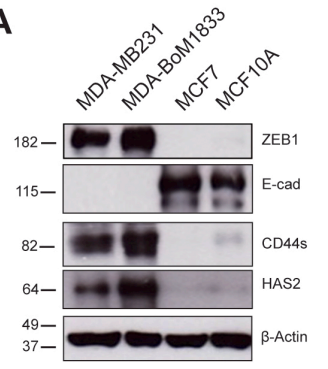

B

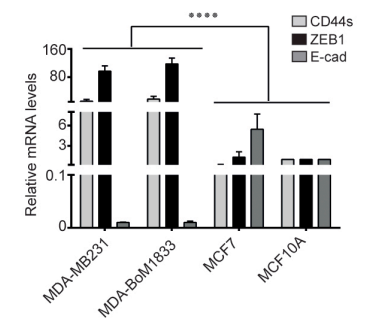

C

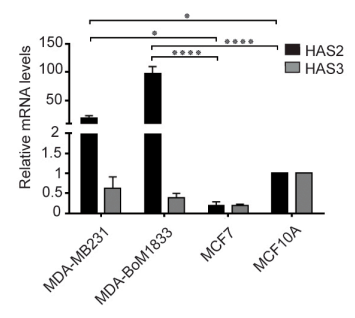

D
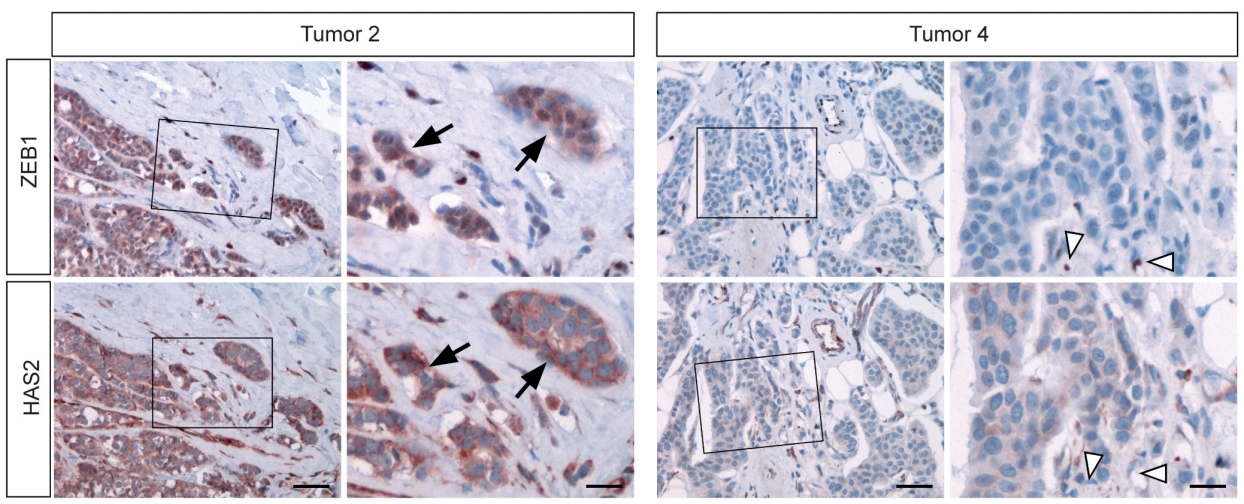

E

CCLE
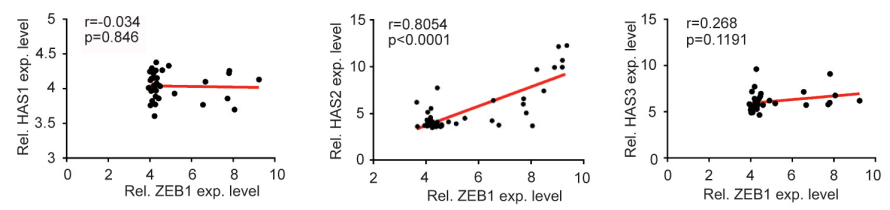

$\mathbf{F}$

Tumors
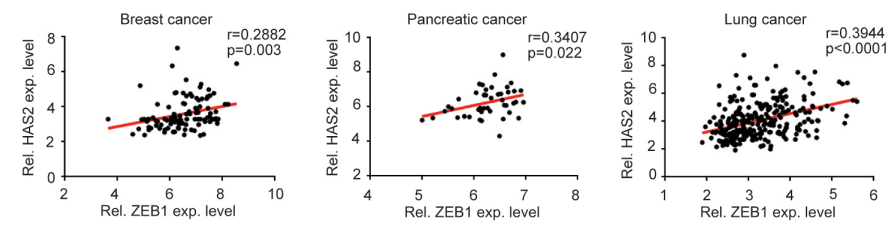

G
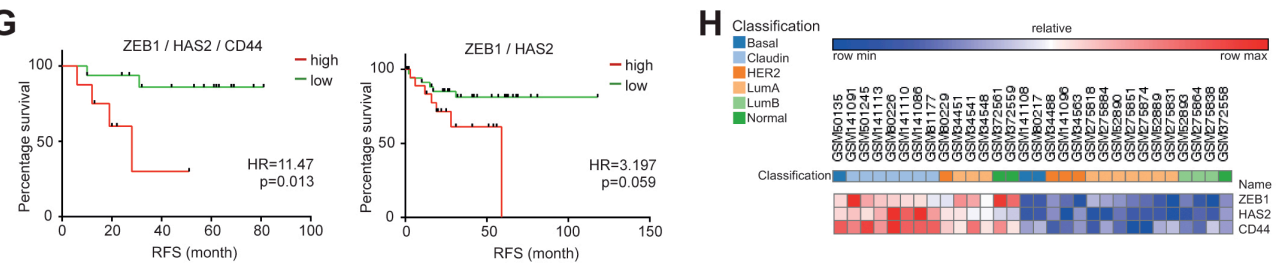

Figure 2: $H A S 2$ correlates with $Z E B B 1$ expression and early relapse in breast cancer. (A) Western blot of MDA-MB231, MDA-BoM1833, MCF7 and MCF10A cell lines. HAS2, CD44s and ZEB1 are coexpressed in mesenchymal-like cancer cells, whereas inversely only E-cad is expressed in the epithelial cell lines. (B, C) mRNA analysis of CD44s, ZEB1 and E-cad (B) and HAS2 and HAS3 (C) in the four cell lines. (D) Immunohistochemical staining for ZEB1 and HAS2 on paraffin sections of breast cancer specimen. In ZEB1positive tumors, areas of tumor cells that express ZEB1 are also positive for HAS2 (Tumor 2, arrows). In tumors without ZEB1 expression, tumor cells show absent or weak expression of HAS2 (Tumor 4). ZEB1-positive stroma cells (open arrowheads) are either HAS2 positive or negative. Scale bars, $50 \mu \mathrm{m}$ (left panel), $20 \mu \mathrm{m}$ (right panel). (E) Correlation analysis of microarray expression data from breast cancer cell lines ('CCLE panel', GSE36133). ZEB1 correlates with HAS2, but not with HAS1 and HAS3 expression. (F) Correlation analysis of microarray expression data from tumor patients. ZEB1 levels are correlated with HAS2 in breast cancer (GSE42568), but also in pancreas (GSE28735) and lung cancer specimens (GSE41271). Pearson correlation coefficients $r$ and $p$-values were computed and are indicated. (G) Kaplan-Meier plots of relapse-free (RFS) survival of upper $58 \%$ ile and lower $42 \%$ ile of combined high and low expression of HAS2, $C D 44$ and ZEB1 (left) and of HAS2 and ZEB1 (right), derived from a microarray and follow-up study collection of 337 tumor samples (GSE18229). Increased ZEB1/HAS2/CD44 and to lesser extend increased ZEB1/HAS2 levels are correlated with early relapse. Hazard ratios (HR) and logrank $p$-values are given. (H) Heat map of expression of ZEB1, HAS2 and CD44 in patient samples in (G). 
changed also the shape of the cells to a cobblestone-like morphology and formation of epithelial clusters, whereas siCtrl transfection did not affect formation of a spindleshape morphology and single cell spreading (Figure 3F).
Similar effects were observed upon siZEB1 and siCD44s transfections. These results indicate that both ZEB1 and HAS2 are essential for TGF $\beta$-induced EMT and their expression is co-regulated, presumably interdependent.
A

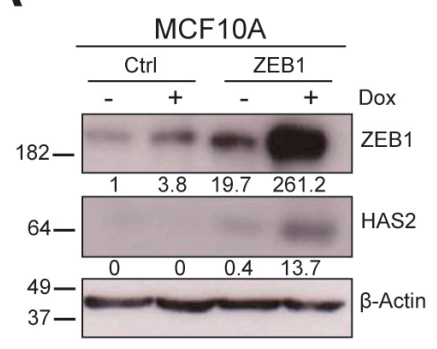

B

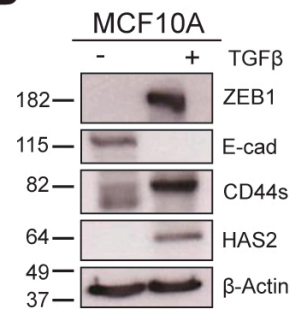

D

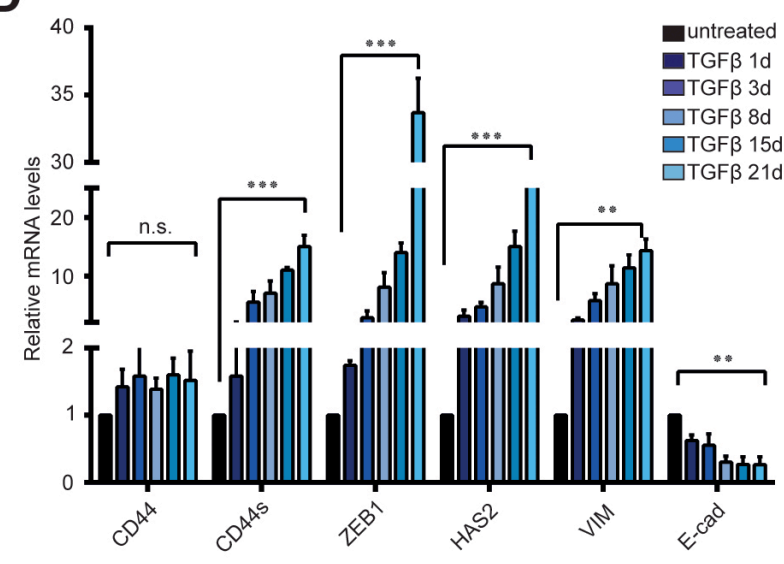

$\mathbf{E}$

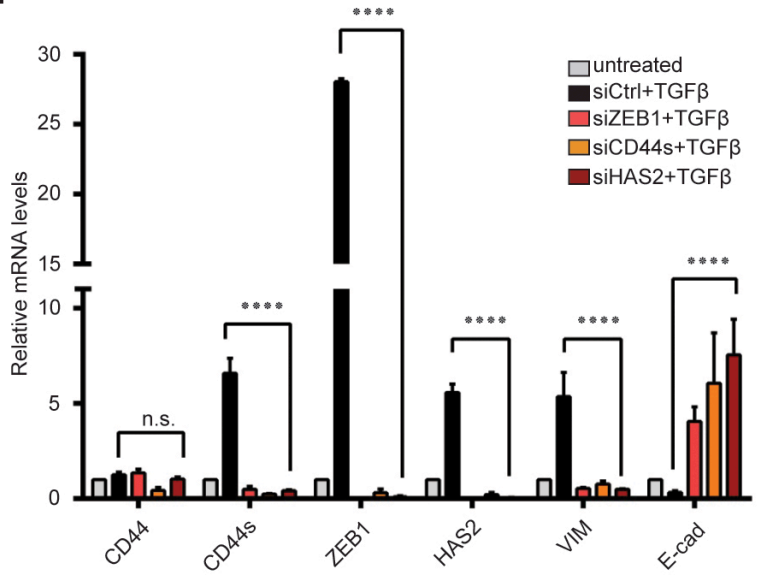

c

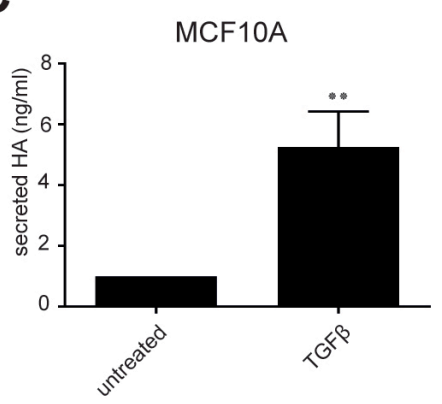

$\mathbf{F}$
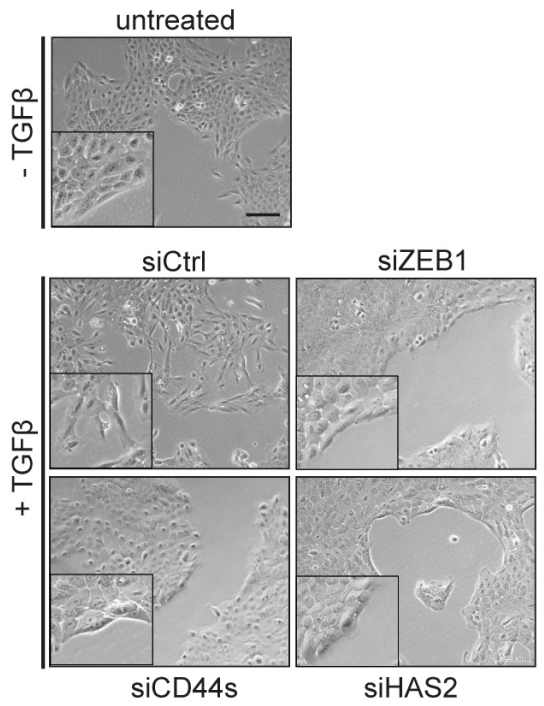

Figure 3: HAS2 becomes activated during EMT along with common EMT markers. (A) MCF10A cells stably transfected with a tetracycline-responsive construct for inducible ZEB1 expression, treated with $1 \mu \mathrm{g} / \mathrm{ml}$ doxycycline (Dox) for six days. Western blot shows induced ZEB1 and upregulation of HAS2 upon Dox treatment. Quantification of blots are given below the individual blots, showing a $>10$-fold upregulation of ZEB1 in 'ZEB1' cells upon Dox treatment as well as a weak Dox-dependent activation of endogenous ZEB1 in 'Ctrl' cells. (B) EMT induction of wildtype MCF10A cells by TGF $\beta$ treatment for 21 days analyzed by Western blot. E-cad is downregulated while CD44s and HAS2 are activated. (C) Treatment of MCF10A cells with TGF $\beta$ increases secretion of HA. (D) Time-course experiment of TGF $\beta$ treatment of MCF10A cells shows a gradual increase of HAS2 levels that follow the increase in ZEB1, vimentin and $C D 44 s$ transcripts by qRT-PCR analysis. Simultaneously, E-cad is downregulated and CD44 total levels remain unchanged. (E) Knockdown of HAS2 prevents EMT in TGF $\beta$-treated MCF10A cells. qRT-PCR of transcript levels upon 21-days TGF $\beta$ treatment and simultaneous knockdown of ZEB1, CD44s or HAS2. Similar to siZEB1 and siCD44s transfection, siHAS2 prevents activation of vimentin, $C D 44 s$ and ZEB1 and E-cad is even increased in comparison to siCtrl samples. (F) Phenotypically, cells are prevented from EMT and stay clustered in all transfectants, except for siCtrl samples. Scale bar, $200 \mu \mathrm{m}$. 


\section{$\mathrm{ZEB} 1$ is activating $H A S 2$ expression by binding to the $H A S 2$ promoter}

ZEB1 regulates various genes, like miR-200 family members, ESRP1 and others in feedback loops [12, 14, 19]. To assess whether $H A S 2$ expression is also directly controlled by ZEB1, we analyzed HAS2 levels upon ZEB1 knockdown and ZEB1 occupancy at the HAS2 locus. In stable shZEB1 knockdown clones of MDA-MB231 cells [42] HAS2 expression was decreased $>100$-fold, whereas that of $H A S 3$ was 5-fold increased. HAS1 was not detected in shGFP or shZEB1 knockdown cells (Figure 4A). The decrease in HAS2 levels was confirmed by Western blotting (Figure 4B). To distinguish between short and long-term effects, we transiently knocked down ZEB 1 in wildtype MDA-MB231 and MDA-BoM1833 cells and observed a similar reduction of HAS2, combined with E-cad upregulation upon ZEB1 knockdown (Figure 4C). Simultaneous to the loss of HAS2, secreted HA was reduced upon siZEB1 transfection in MDA-MB231 and MDA-BoM1833 cells (Figure 4D). Vice versa, overexpression of ZEB1 in the epithelial MCF7 breast cancer cell line resulted in downregulation of E-cad and a slight activation of HAS2 (Figure 4E). To understand whether ZEB1 directly binds to the HAS2 promoter, we cloned a -2000 to +1 bp fragment of the human HAS2 locus upstream of a luciferase reporter gene. Knockdown of ZEB1 in MDA-MB231 and MDA-BoM1833 cells resulted in reduced activity of the HAS2-luciferase reporter construct (Figure 4F). A closer inspection of the promoter region identified eight E-boxes as putative binding sites for ZEB1 in this construct. Five of them were located between -2000 and $-1000 \mathrm{bp}$ and three were identified at positions $-329,-522$ and $-563 \mathrm{bp}$ (Figure $4 \mathrm{G})$. We generated deletion constructs del1 and del2 that harbor the three proximal E-boxes and no E-boxes, respectively (Figure 4G). In MCF7 cells the full-length construct revealed a 3-fold activation upon transient ZEB1 overexpression (Figure 4H). This effect was reduced if regions harboring five or all eight E-boxes were deleted. However, a moderate 2-fold upregulation of the luciferase reporter was observed with the del2 construct that was not completely diminished in the empty vector control (Figure 4H). As we have shown previously that ZEB1 is interacting with YAP to activate specific common target genes [9], HAS2 might be activated by a similar E-box independent mechanism. Chromatin immunoprecipitation of the endogenous HAS2 locus in MDA-MB231 cells identified a substantial enrichment of ZEB1 at the promoter region of HAS2 $(-465 \mathrm{bp})$ in contrast to a random distal region at -4500 bp (Figure 4I). This enrichment was lost in ZEB1 knockdown cells, similar to the known ZEB1 target gene EPCAM (Figure 4J). However, we could not identify a specific YAP binding to the HAS2 locus, as YAP knockdown did not result in decreased ZEB1-mediated precipitation of the HAS2 promoter (Figure 4J) and anti-YAP ChIP did not show enrichment in shCtrl vs. shYAP cells (Figure 4K). Interestingly, HAS2 levels were reduced 2-fold upon YAP knockdown, although not significantly (Supplementary Figure S5). These results indicate that HAS2 expression which is the main driver of HA production in the analyzed breast cancer cell lines is directly controlled by ZEB1, thereby linking initial EMT signals to the secretion of excess HA.

\section{Extracellular HA and conditioned medium of MDA-MB231 and MDA-BoM1833 cells accelerates osteoclast differentiation in a HAS2- dependent manner}

The life-threatening event during tumor progression downstream of EMT is the formation of metastases. It was previously shown that HAS2 is crucial for creating a prometastastic microenvironment allowing triple-negative breast cancer cells to efficiently colonize to the bone $[34,43]$. This is in part attributed to enhanced osteolysis by increased osteoclast differentiation which is stimulated by HAS2 and HA [44]. We wondered whether the ZEB1-dependent induction of HAS2 and thus increased HA secretion enhances osteoclasts differentiation in support of formation of a pro-metastatic niche. Therefore we used murine monocyte-macrophagic Raw264.7 cells, a well-established in vitro model of osteoclast differentiation. In presence of RANKL Raw264.7 cells start to form osteoclasts within one week, identified by tartrate-resistant acid phosphatase (TRAP) staining (Figure 5A and 5B). When these cells were incubated with HA in addition to RANKL, the amount of differentiated osteoclasts was more than doubled from 60 to 130 in a 6-well plate, indicating that external supply of HA is promoting osteoclast differentiation (Figure 5A and 5B). Importantly, when Raw264.7 cells were incubated with supernatant of MDA-MB231 or MDA-BoM1833 cells in which ZEB1 was knocked down, the amount of osteoclasts was significantly reduced to $10 \%$ in comparison to incubation with control supernatant. A similar but less pronounced effect was observed when $C D 44 s$ or HAS2 expression was silenced by siRNA in MDA-MB231 or MDA-BoM1833 cells (Figure 5C and $5 \mathrm{D}$ ). These results demonstrate that tumor cell derived HA promotes osteoclast differentiation and that the effect is dependent on HAS2, CD44s and ZEB1 activities.

\section{DISCUSSION}

Besides the deregulation of key signaling pathways, tumor progression is dependent on the ability of tumor cells to recruit and instruct the microenvironment for providing pro-tumorigenic cues. These cues are required to promote tumor growth, survival, evasion from immune surveillance and metastatic spread by EMT induction [21]. The interaction with the microenvironment involves remodeling 
A

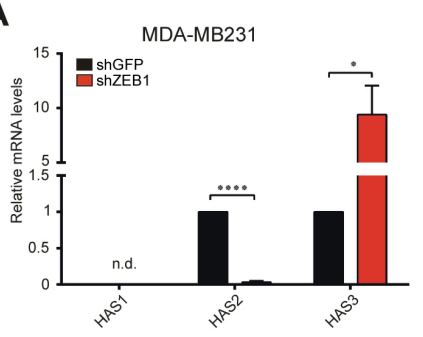

D
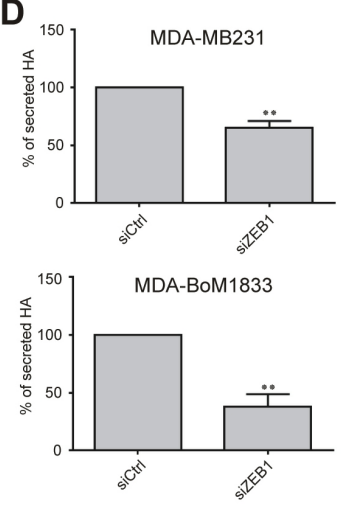

G

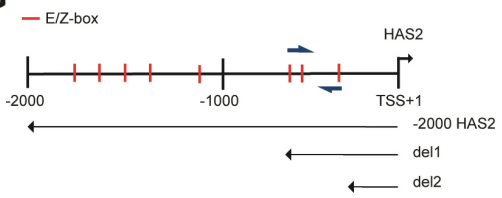

B

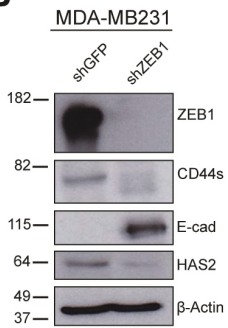

E

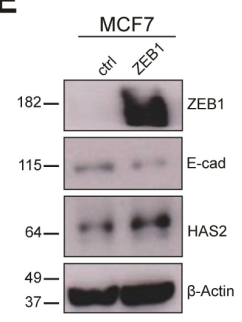

C
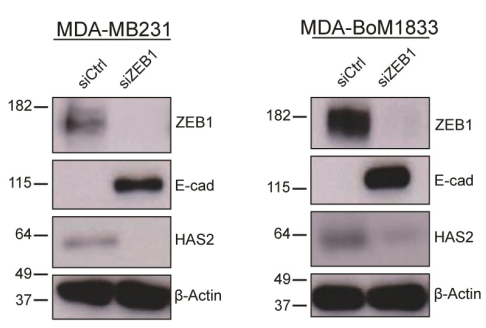

F
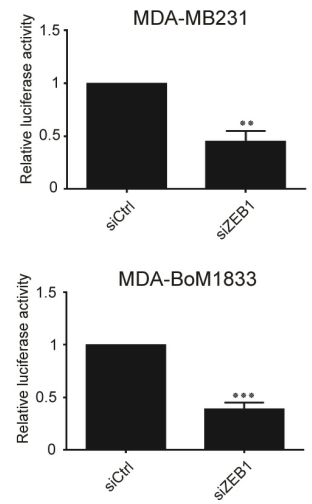

H

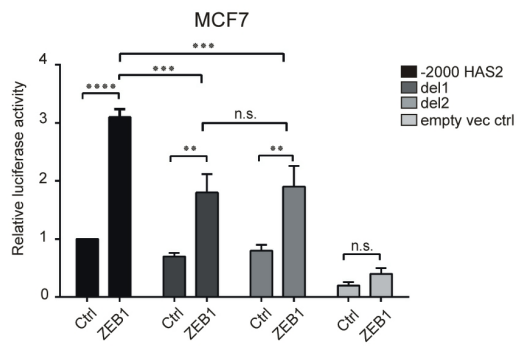

I

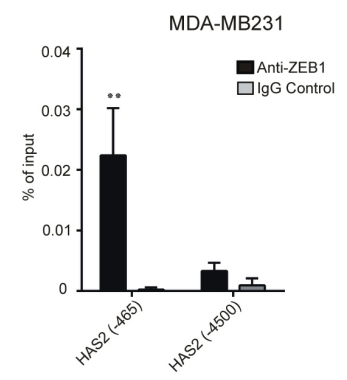

J

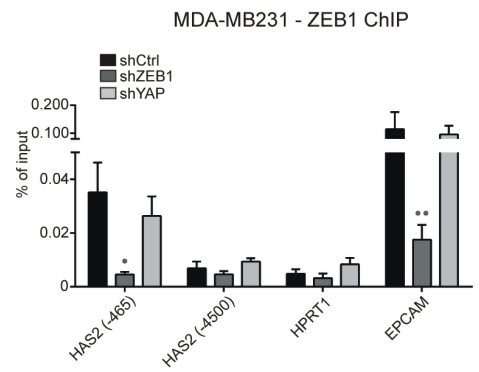

K

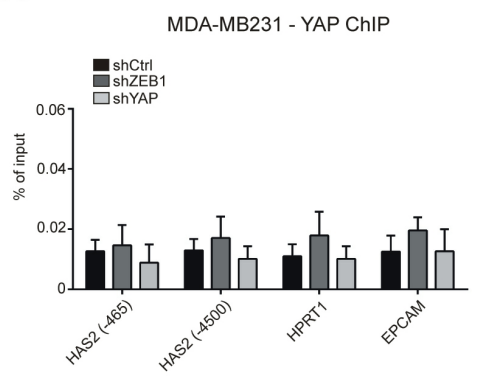

Figure 4: ZEB1 directly regulates HAS2 expression. (A) mRNA analysis of HAS1, HAS2 and HAS3 levels in MDA-MB231 cells after stable ZEB1 knockdown shows a specific loss of HAS2 transcripts whereas HAS3 is even upregulated. HAS1 is not detected (n.d.) in shGFP and shZEB1 samples. (B) Western blot analysis of a stable knockdown of ZEB1 in MDA-MB231 cells. (C) Western blot of cells with a transient knockdown of ZEB1 in MDA-MB231 and MDA-BoM1833 cells. Knockdown of ZEB1 substantially reduces HAS2 expression. (D) Transient ZEB1 knockdown reduces levels of secreted HA in MDA-MB231 and MDA-BoM1833 cells. (E) Overexpression of ZEB1 in MCF7 cells induces HAS2 upregulation and E-cad reduction observed by Western blot. (F) Luciferase assay in MDA-MB231 and MDABoM1833 cells transiently transfected with a -2000 bp to +1 bp promoter fragment of the human HAS2 locus cloned 5-prime of a luciferase reporter gene. Knockdown of ZEB1 reduces the reporter gene activity. (G) Schematic representation of the human HAS2 promoter region between -2000 and +1 bp relative to the transcription start site (TSS). Red lines indicate positions of canonical E- and Z-boxes and primer locations used for ChIP are indicated by blue arrows. Sequences included in deletion constructs containing three proximal E-boxes (del1) and no E-boxes (del2) are indicated by arrows. (H) Transient transfection of MCF7 cells with the full-length HAS2 promoter construct, del1 or del2 reveal that co-transfected ZEB1 is activating the full-length construct and to lesser extend the two deletion constructs. (I) Chromatin immunoprecipitation (ChIP) using anti-ZEB1 and control IgG in MDA-MB231 cells. Enrichment of ZEB1 is seen at the proximal promoter (-465), but not at a distal region of the locus $(-4500)$. ( $\mathbf{J}, \mathbf{K})$ ChIP of the HAS2 promoter in MDA-MB231 shCtrl, shZEB1 and shYAP stable knockdown cells with anti-ZEB1 (J) and anti-YAP $(\mathrm{K})$ antibodies, showing that ZEB1-specific DNA precipitation is lost at HAS2 (-465), similar to the promoter of the known ZEB1 target gene EPCAM, whereas ZEB1 binding is unaffected by YAP knockdown. 
of the extracellular matrix, including increased deposition of different proteoglycans such as hyaluronic acid into the extracellular space [20]. We found tumor cell-derived HA to promote EMT, presumably by binding to CD44s. While excess HA induced $Z E B 1$ expression, blocking HA synthesis reduced $Z E B 1$ levels in triple-negative breast cancer cells. Expression of ZEB1 and $H A S 2$, the main synthase of HA in tumor cells, was strongly correlated in breast cancer cell lines and tumor patient samples and high expression levels of $H A S 2$ were associated with poor survival. Interestingly, ZEB1 was found to bind to the HAS2 promoter and to activate its expression. This suggests another level of maintaining an EMT state of tumor cells by enforcing the autocrine HA production via ZEB1-mediated transcriptional activation of $H A S 2$. HA secretion by tumor cells affected not only tumor cells but also the pro-metastatic niche. We found that conditioned medium from MDA-MB231 and even stronger from the selected bone-metastatic subline MDA-BoM1833 enhanced the differentiation of monocytes to osteoclasts. This is of particular interest for the formation of macrometastases as tumor cells have to remodel the bone for efficient colonization $[45,46]$. Accordingly, tumor cells-derived HA likely interferes with bone homeostasis by shifting the equilibrium of bone generating osteoblasts and osteolytic osteoclasts towards increased osteoclast numbers supporting bone destruction. In line with our findings, HAS2 was shown to promote tumor growth and metastases in bones by stimulating the interaction of breast cancer stem-like cells with macrophages and stromal cells [43]. Similarly, inactivating CD44 as the main HA receptor by shRNA knockdown blocked metastatic spreading of MDA-MB231 cells injected into the mammary fat pad of immunodeficient mice. The reduction of bone metastases correlated with a decrease in the number of osteolytic osteoclasts [44].

In the differentiation experiment of Raw264.7 cells conditioned medium of MDA-MB231 or MDA-BoM1833 cells with a ZEB1 knockdown reduced osteoclast differentiation more efficiently than knockdown of HAS2 and CD44. ZEB1 was shown to induce the expression of the BMP inhibitors NOG, FOL and CHRDL1, which are secreted and known to promote osteolysis [11, 47]. Hence, HA and BMP inhibitors cooperate in bone remodeling. Accordingly, knockdown of ZEB1 in the in vitro differentiation system had a stronger effect, because it is blocking HAS2 expression and HA secretion and is reducing $\mathrm{BMP}$-inhibitor accumulation in the conditioned medium simultaneously.

HA synthesis by HAS1-3 and its deposition into the ECM is increased during EMT and is known to play a
A

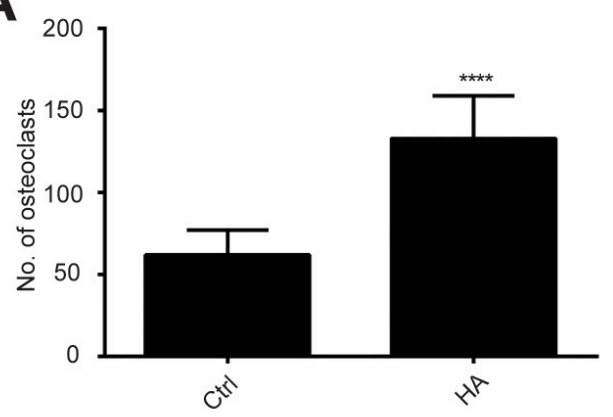

C

MDA-MB231

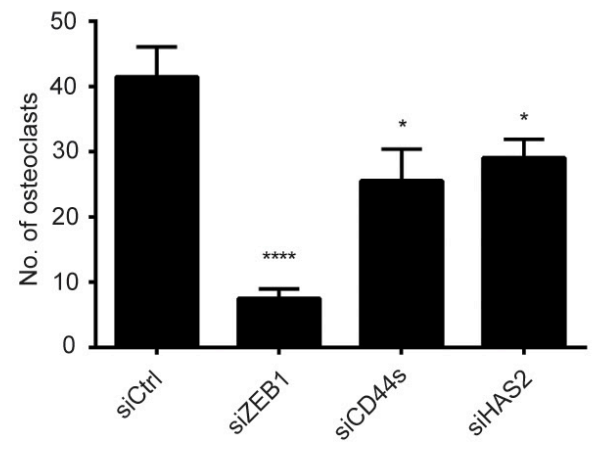

B

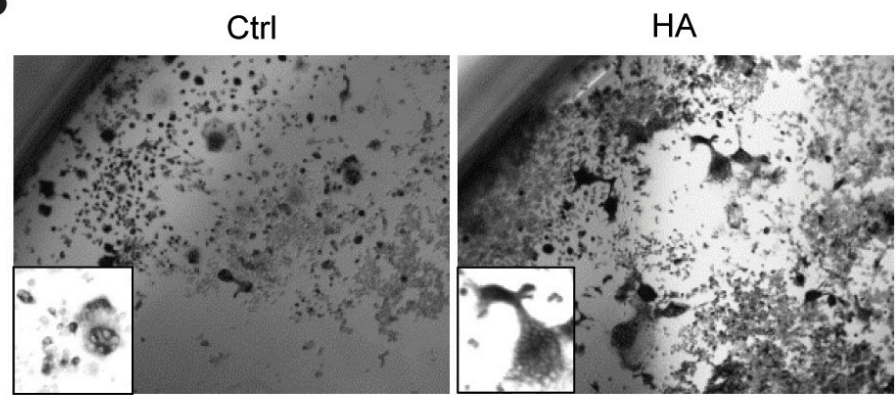

D

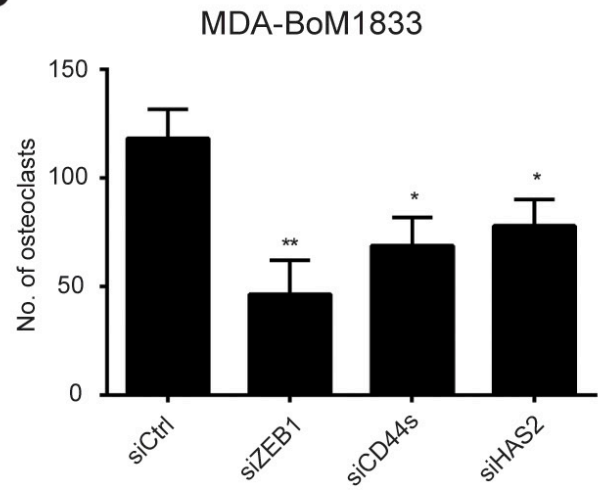

Figure 5: HA-enriched conditioned medium of MDA-MB231 and MDA-BoM1833 cells induces osteoclast differentiation of Raw264.7 cells to osteoclasts. (A, B) Quantification (A) and morphology (B) of tartrate-resistant acid phosphatase stained osteoclasts in cultures of Raw264.7 cells under differentiation conditions with RANKL (Ctrl). Differentiation is enhanced 2.5 fold by additional HA treatment (HA). (C, D) Conditioned medium of MDA-MB231 (C) and MDA-BoM1833 cells (D) is providing differentiation cues for osteoclast formation of Raw264.7 cells. Knockdown of ZEB1, CD44s and HAS2 reduces the capacity of the conditioned medium to induce osteoclast differentiation. 
crucial role during tumor progression [20]. Recently, the corresponding synthase genes in mice, in particular Has 2 and Has3, were found among the most highly upregulated genes during the early phase of TGF $\beta$-induced EMT. This was dependent on Smad4 and the tumor suppressive function of TGF $\beta$ in a mouse model of pancreatic cancer [48]. These findings support our idea that HAS2 is induced during EMT by ZEB1 and that its production of HA is crucial for sustaining EMT signals.

Of note, it was demonstrated that HAS2 supports tumorigenesis mainly by its synthase activity of HA [20]. However, an HA-independent function of HAS2 supporting tumor progression was suggested by recent findings. Blocking newly synthesized HA or blocking HA-CD44 interaction did not efficiently block EMT in TGF $\beta$-treated NMuMG, whereas knockdown of Has 2 completely abolished EMT [49]. Whether an intracellular HA-synthase-independent function of HAS2 is active in our cellular models could not be sufficiently addressed. However, we observed strong effects on ZEB1 levels and EMT when HA synthesis was blocked by 4-MU. In line with this, CD44s seems to be required for the effect of HA on ZEB1 expression, but how HA and CD44s then mechanistically act on $Z E B 1$ transcription remains elusive. Very likely, HA binds to CD44s which then induces signaling by the receptor [26].

Although our results and several other studies indicate a pro-tumorigenic function of $\mathrm{HA}$, there are also conflicting data that support a more anti-tumorigenic function. In squamous cell carcinoma decreased HA levels were associated with poor survival. Similarly, in cutaneous melanoma reduced HA and CD44 levels lead to an early tumor relapse and poor survival $[50,51]$. These effects might be related to different functions of low, medium and high molecular weight HA in a context-dependent manner. As an example: high molecular weight HA acts mainly anti-angiogenic as it inhibits endothelial proliferation and migration in vitro [20]. However, in vivo experiments showed that it supports angiogenesis presumably by interacting with different ECM components like FGFs and proteoglycans [52]. This shows that the function of HA is very complex. Moreover, the balance between HA synthesis and degradation controls the cellular responses. It will be interesting to further explore on this equilibrium in breast cancer to obtain a comprehensive view on how HA triggers EMT, invasion and metastasis.

Recent findings support the notion that ZEB1 not only acts as a transcriptional repressor to suppress epithelial-specific genes like $E$-cad and members of the miR-200 family, but can also activate transcription in specific contexts by interacting with different co-factors $[9,11]$. Interestingly, this function of ZEB1 does not necessarily require direct binding of ZEB1 to the DNA. The activation of a specific ZEB1/YAP target gene set seems to be independent of any canonical ZEB1 DNAbinding motif, like $\mathrm{Z}$ - or E-boxes at the target gene promoters [9]. In our analysis, a reduced expression of a $H A S 2$ luciferase reporter construct was detected when proximal E-box motifs were deleted. However, ZEB1 was still able to partially activate the construct in absence of a conserved E-box. Although we have not formally proven that ZEB1 is not directly interacting with the DNA of the $H A S 2$ promoter, a similar mechanism as for common ZEB1/YAP target genes may be active to drive HAS2 expression. It will be interesting to further dissect how ZEB1 is activating the promoter and which transcription factors are involved.

In summary, our results provide novel insights into how ZEB1 utilizes HAS2/HA to enforce its own expression and to shape the microenvironment. This crosstalk is used as amplifying module to support the previously identified ZEB1/ESRP1/CD44s feedback loop [19]. Initial external EMT-stimuli activate $Z E B 1$ expression that simultaneously provides ligand (HA) and generation of the corresponding receptor (CD44s) to further accelerate EMT. Activation of (1) CD44 differential splicing by ESRP1-loss and (2) activation of HA synthesis by direct regulation of HAS2 help to maintain high $Z E B 1$ expression. In conclusion, together with our previous findings, the analysis provides insights into a complex multi-factorial feedback system controlled by ZEB1 to induce EMT and metastatic behavior of breast cancer cells.

\section{MATERIALS AND METHODS}

\section{Cell culture}

MDA-MB231, MCF7, MCF10A and monocytemacrophagic Raw264.7 cell lines were purchased from ATCC and MDA-BoM1833 were kindly provided by Joan Massagué (Sloan-Kettering Institute for Cancer Research, New York). MDA-MB231 shGFP and shZEB1 stable knockdown clones have been described previously [42] and MDA-MB231 shCtrl and shYAP knockdown cells were generated by lentiviral transduction of pGIPZ constructs harboring a non-silencing control (RHS4346) and V3LHS-306099, respectively. Non-transduced cells were eliminated by puromycin selection $(2.5 \mu \mathrm{g} / \mathrm{ml})$ for 3 days. Cells were cultured in DMEM/10\% FCS or in DMEM/F12 (Invitrogen, 10566 and 31331) supplemented with 5\% horse serum (Life Technologies, 16050122), $20 \mathrm{ng} / \mathrm{ml}$ EGF (R\&D Systems, 236EG200), $0.5 \mu \mathrm{g} / \mathrm{ml}$ hydrocortisone (Sigma, H0888), $0.1 \mu \mathrm{g} / \mathrm{ml}$ cholera toxin (Sigma, C-8052) and $10 \mu \mathrm{g} / \mathrm{ml}$ insulin (Invitrogen, 12585014) for MCF10A cells. EMT was induced by treatment with $5 \mathrm{ng} / \mathrm{ml}$ TGF $\beta 1$ (PeproTech, 100-21) for the indicated time replacing the medium every other day. Induction of ZEB1 expression in MCF10A cells was induced by adding $1 \mu \mathrm{g} / \mathrm{ml}$ doxycycline (Dox, Sigma, D9891) every other day as described previously $[19,53]$. Inhibition of HAS1-3 to block HA synthesis was performed by treatment of cells 
with indicated concentrations of 4-Methylumbelliferone (4-MU, Sigma, M1381) for 72 h. Hyaluronic acid sodium salt from Streptococcus equi bacterial glycosaminoglycan polysaccharide (HA, Sigma, 53747-1G) or Sodium Hyaluronate 5000 (Healon, 10-2000-12) was dissolved in $\mathrm{H}_{2} \mathrm{O}$ and used at a final concentration of $250-400 \mu \mathrm{g} / \mathrm{ml}$ in DMEM $/ 10 \% \mathrm{FCS}$ for $24 \mathrm{~h}$. All cells were kept at $37^{\circ} \mathrm{C}$, $5 \% \mathrm{CO}_{2}$ in a humidified incubator.

\section{Western blotting}

Cells were rinsed once in PBS and lysed in TLB. $30 \mu \mathrm{g}$ of protein was separated by SDS-PAGE (10\%) for $1 \mathrm{~h}, 150 \mathrm{~V}$ and transferred to a nitrocellulose membrane by wet blotting in transfer buffer for $2 \mathrm{~h}, 300 \mathrm{~mA}$ at $4^{\circ} \mathrm{C}$. Membranes were immersed in Antigen pretreatment solution (SuperSignal Westernblot Enhancer, Thermo Scientific) for $10 \mathrm{~min}$ and blocked in 5\% skim milk/ TBST) for $30 \mathrm{~min}$ at room temperature. Primary antibody incubation was carried out in Primary antibody Diluent (SuperSignal Westernblot Enhancer, Thermo, 46641) over night at $4^{\circ} \mathrm{C}$. After washing in TBST, membrane was incubated with HRP-conjugated secondary antibody in $5 \%$ skim milk/TBST for $1 \mathrm{~h}$ at RT. Detection was carried out using SuperSignal West Femto Maximum Sensitivity Substrate (Thermo, 34094) or ECL Prime Westenblot Detection Reagent (Amersham, RPN2232) and a ChemiDoc imaging system (BioRad). Quantification was performed where appropriate using ImageJ and presented normalized to $\beta$-Actin levels.

\section{Antibodies}

The following antibodies and dilutions were used for Western blotting: mouse anti-E-cadherin (BD Pharmingen, $610181 ; 1: 5,000)$, mouse anti- $\beta$-Actin (Sigma, A5441; 1:5,000), mouse anti-CD44 (R\&D Systems, BBA10; 1:1000), mouse anti-HAS2 (Abcam, H00054845-B01P; 1:500) and rabbit anti-ZEB1 (Sigma, HPA027524; $1: 5,000)$, as well as HRP-coupled goat anti-rabbit IgG (Dianova, 111-035-003; 1:25,0000) and goat anti-mouse IgG (Dianova, 115-035-003; 1:25,000).

\section{Total RNA isolation and quantitative reverse transcriptase PCR (qRT-PCR)}

Total RNA was isolated using the RNeasy Plus Mini Kit (Qiagen, 74136) according to manufacturer's protocol. cDNA was synthesized from $1 \mu \mathrm{g}$ total RNA with the RevertAid First Strand cDNA Synthesis Kit (Thermo, K1622), using $0.5 \mu$ l oligo (dT) and $0.5 \mu \mathrm{l}$ random hexamer primers. Transcript levels were analyzed by qRT-PCR using cDNA amounts corresponding to $7.5 \mathrm{ng}$ of original total RNA and $300 \mathrm{nM}$ primers (see Supplementary Table S1) with the Power SYBR Green PCR Master Mix (Applied Biosystems, 4368702) in a
LightCycler 480 (Roche). Relative expression levels were calculated and normalized to those of $A C T B$ applying the Pfaffl method.

\section{Indirect immunofluorescence labeling}

Cells were plated on cover slips, fixed with $1 \%$ PFA/PBS, followed by permeabilization step with $0.25 \%$ Triton X-100/PBS (10 min each). After blocking in 3\% BSA/PBS for $30 \mathrm{~min}$, antibodies were diluted 1:200 in blocking solution and applied for incubation overnight. Alexa488 and -594 conjugated antibodies were used in same dilutions for 45-60 min. Cover slips were mounted with Citifluor/1 $\mu \mathrm{g} / \mathrm{ml}$ DAPI and imaged on a Leica DM5500 microscope.

\section{Generation of reporter gene constructs and luciferase assay}

$2 \mathrm{~kb}$ of the HAS2 promoter region containing E-Boxes at $-329,-522,-563,-1065,-1397,-1508$, -1613 and -1743 bp as well as del1 ( -702 to $+1 \mathrm{bp})$ and del2 $(-248$ to +1$)$ sequences were amplified by PfuUltra HF polymerase (Agilent, 600380-51) with forward1 (5'ataatgagctcaacaacaaatgtgtttttct-3'), forward 2 (5'-ataatgag ctcccacggcagaaacctcttta-3') or forward3 (5'-ataatgagctccg gcctgtagctcagagaag- $\left.3^{\prime}\right)$ and reverse ( $5^{\prime}$-ataatagatcttccttcc ccgecgttgttgc- $\left.3^{\prime}\right)$ primers and cloned $5^{\prime}$ of the luciferase gene into pGL4.10 (Promega) by XhoI/XbaI using standard molecular cloning techniques. For the luciferase reporter assay, cells were seeded in 24-well plates and transfected $24 \mathrm{~h}$ later with siRNAs (see Supplementary Table S2) and with plasmid DNA another $48 \mathrm{~h}$ later. The second transfection contained $50 \mathrm{ng}$ pRL-TK, $300 \mathrm{ng}$ luciferase reporter construct and $150 \mathrm{ng}$ pCIneo-ZEB1 or pCIneo empty vector control. Cells were harvested 24 $\mathrm{h}$ after plasmid transfection and lysed in $100 \mu \mathrm{l}$ passive lysis buffer for $15 \mathrm{~min}$ and measured by using the DualLuciferase $^{\circledR}$ Reporter Assay System (Promega, E1910) according to the manufacturer's instructions. Firefly luciferase levels were normalized to Renilla luciferase levels and the activity of the $-2000 H A S 2$ promoter construct alone was set to one. Experiments were repeated three times.

\section{Transfection of plasmid DNA and siRNA}

Plasmid DNA transfection was done by using the FugeneHD transfection reagent (Promega, E2311) according to the manufacturer's instructions. siRNAs were purchased from Ambion and sequences are provided in Supplementary Table S2. $0.4 \mathrm{mM}$ of individual siRNAs were transfected with Lipofectamine RNAiMax (Invitrogen, 13778075) and unless otherwise indicated harvested $72 \mathrm{~h}$ afterwards for protein or RNA analysis as previously described [19]. 


\section{Chromatin immunoprecipitation}

ChIP was performed as previously described [11]. $500 \mu \mathrm{g}$ of chromatin was incubated with $5 \mu \mathrm{g}$ of anti-ZEB1 (Santa Cruz, H102, sc-25388X) and rabbit IgG control (Santa Cruz, sc-2345) antibodies at $4^{\circ} \mathrm{C}$ overnight. $25 \mu \mathrm{l}$ of a 1:1 ratio of protein $\mathrm{A} / \mathrm{G}$ Dynabeads (Invitrogen, 10002D, 10004D) were used to precipitate antibody-bound chromatin. Upon elution with $0.1 \mathrm{M}$ NaHCO3, 1\% SDS and decrosslinking with $250 \mu \mathrm{g} / \mathrm{ml}$ RNase A and $500 \mu \mathrm{g} / \mathrm{ml}$ proteinase $\mathrm{K}$ at $65^{\circ} \mathrm{C}$ overnight, specific genomic regions were analyzed by qPCR. Primers are given in Supplementary Table S3.

\section{Statistical analysis}

Statistical analyses were performed using GraphPad Prism 6 (GraphPad Software, Inc.). Normalized relative expression levels were used to calculate the mean and the SEM for $n=3$ unless otherwise stated. Statistical significance was evaluated by two-tailed Student's $t$-test and multi-group comparisons by 2-way ANOVA and Tukey's post-hoc test was used when appropriate. $p$-values of statistical significance are given: ${ }^{*} p<0.05$; ${ }^{* *} p<0.01 ; * * p<0.001 ; * * * * p<0.0001$.

\section{Correlation analysis and Kaplan-Meier plot}

Expression data for the 'CCLE panel' of cancer cell lines comprising 36 cancer types from 917 cancer cell lines, the 'NCI60 panel' and of 275 lung, 104 breast and 45 pancreatic cancer patients, including follow-up data, were downloaded from the NCBI GEO database (GSE5846, GSE36133， GSE41271， GSE42568, GSE28735, GSE2034, GSE18229) [35-39, 54, 55]. Correlation analysis was done using GraphPad Prism. Relapse and overall survival was compared between patients with high and low HAS2 expression levels with a cut-off at the average expression values above the 58th percentile and below the 42th percentile. Kaplan-Meier plotting and logrank test was used to evaluate the differences in survival between defined groups.

\section{Immunohistochemistry}

Samples were retrieved from local archives and usage was approved by the Ethics Committee of the University of Freiburg. Immunohistochemistry was performed as described previously [9] using paraffin embedded tissue, sectioned to $4 \mu \mathrm{m}$, anti-ZEB1 (Sigma, \#HPA027524; 1:800), anti-HAS2 (Abcam, H00054845-B01P; 1:200) antibodies and the EnVision-System (DAKO, K4003, K4001). Antigen retrieval was carried out on deparaffinized and rehydrated sections in $10 \mathrm{mM}$ citrate buffer, $\mathrm{pH} 6.0$ in a pressure cooker and DAB substrate (Thermo, 002020) was used for visualization of antibody binding. Slides were counterstained with Mayer's hematoxylin (Merck, 1.09249.0500) and mounted with Histokitt (Roth, 6640).

\section{Osteoclast differentiation assay}

The secretome of individual cell lines and transfectants was analyzed to promote osteoclast differentiation of Raw264.7 cells as previously described [11]. In brief, supernatant of MDA-MB231 and MDA-BoM1833 transfected with siRNAs was collected after $24 \mathrm{~h}$. One thousand Raw264.7 cells per well were seeded in a 96-well plate and incubated with cell supernatants supplemented with $5 \mathrm{ng} / \mathrm{ml}$ RANKL (PreproTech, 315-11) as indicated with or without $250 \mathrm{ng} / \mathrm{ml}$ hyaluronic acid. Tartrate-resistant acid phosphatase (TRAP) staining was done with the leukocyte acid phosphatase kit (Sigma, 387A-1KT) one week afterwards to access the number of osteoclasts.

\section{Enzyme-linked immunosorbent assay (ELISA)}

The amount of secreted HA was analyzed by Quantikine ELISA Hyaluronan KIT (R\&D, DHYAL0). Cells were serum-starved for $24 \mathrm{~h}$ and medium was collected and processed according to the manufacturer's instructions.

\section{ACKNOWLEDGMENTS AND FUNDING}

We thank Jessica Pfannstiel, Stephanie Mewes, Anja Schmitt and Britta Schlund for excellent technical assistance and Florian Sigloch for helpful discussion. We are grateful to Drs Joan Massagué for providing MDA-BoM1833 cells and Tilman Brummer for MCF10A cells with inducible ZEB1 expression. This work was supported by the German Research Foundation (SFB850 TP A4, B2 and C8, the DKTK initiative (DKFZ), and DFG BR 1399/9-1, DFG 1399/10-1, DFG BR4145/1-1).

\section{CONFLICTS OF INTEREST}

The authors declare no conflicts of interest.

\section{REFERENCES}

1. Brabletz T. To differentiate or not-routes towards metastasis. Nat Rev Cancer. 2012; 12:425-436.

2. Geng SQ, Alexandrou AT, Li JJ. Breast cancer stem cells: Multiple capacities in tumor metastasis. Cancer Lett. 2014; 349:1-7.

3. Polyak K, Weinberg RA. Transitions between epithelial and mesenchymal states: acquisition of malignant and stem cell traits. Nat Rev Cancer. 2009; 9:265-273.

4. Thiery JP, Acloque H, Huang RY, Nieto MA. Epithelialmesenchymal transitions in development and disease. Cell. 2009; 139:871-890. 
5. Nieto MA. Epithelial plasticity: a common theme in embryonic and cancer cells. Science. 2013; 342:1234850.

6. De Craene B, Berx G. Regulatory networks defining EMT during cancer initiation and progression. Nat Rev Cancer. 2013; 13:97-110

7. Peinado H, Olmeda D, Cano A. Snail, Zeb and bHLH factors in tumour progression: an alliance against the epithelial phenotype? Nat Rev Cancer. 2007; 7:415-428.

8. Puisieux A, Brabletz T, Caramel J. Oncogenic roles of EMT-inducing transcription factors. Nat Cell Biol. 2014; $16: 488-494$.

9. Lehmann W, Mossmann D, Kleemann J, Mock K, Meisinger C, Brummer T, Herr R, Brabletz S, Stemmler MP, Brabletz T. ZEB1 turns into a transcriptional activator by interacting with YAP1 in aggressive cancer types. Nat Commun. 2016; 7:10498.

10. Meidhof S, Brabletz S, Lehmann W, Preca BT, Mock K, Ruh M, Schuler J, Berthold M, Weber A, Burk U, Lubbert M, Puhr M, Culig Z, et al. ZEB1-associated drug resistance in cancer cells is reversed by the class I HDAC inhibitor mocetinostat. EMBO Mol Med. 2015; 7:831-847.

11. Mock K, Preca BT, Brummer T, Brabletz S, Stemmler MP, Brabletz T. The EMT-activator ZEB1 induces bone metastasis associated genes including BMP-inhibitors. Oncotarget. 2015; 6:14399-14412. doi: 10.18632/oncotarget.3882.

12. Wellner U, Schubert J, Burk UC, Schmalhofer O, Zhu F, Sonntag A, Waldvogel B, Vannier C, Darling D, zur Hausen A, Brunton VG, Morton J, Sansom O, et al. The EMT-activator ZEB1 promotes tumorigenicity by repressing stemnessinhibiting microRNAs. Nat Cell Biol. 2009; 11:1487-1495.

13. Mani SA, Guo W, Liao MJ, Eaton EN, Ayyanan A, Zhou AY, Brooks M, Reinhard F, Zhang CC, Shipitsin M, Campbell LL, Polyak K, Brisken C, et al. The epithelialmesenchymal transition generates cells with properties of stem cells. Cell. 2008; 133:704-715.

14. Burk U, Schubert J, Wellner U, Schmalhofer O, Vincan E, Spaderna S, Brabletz T. A reciprocal repression between ZEB1 and members of the miR-200 family promotes EMT and invasion in cancer cells. EMBO Rep. 2008; 9:582-589.

15. Gregory PA, Bert AG, Paterson EL, Barry SC, Tsykin A, Farshid G, Vadas MA, Khew-Goodall Y, Goodall GJ. The miR-200 family and miR-205 regulate epithelial to mesenchymal transition by targeting ZEB1 and SIP1. Nat Cell Biol. 2008; 10:593-601.

16. Park SM, Gaur AB, Lengyel E, Peter ME. The miR-200 family determines the epithelial phenotype of cancer cells by targeting the E-cadherin repressors ZEB1 and ZEB2. Genes Dev. 2008; 22:894-907.

17. Zanconato F, Cordenonsi M, Piccolo S. YAP/TAZ at the Roots of Cancer. Cancer Cell. 2016; 29:783-803.

18. Cordenonsi M, Zanconato F, Azzolin L, Forcato M, Rosato A, Frasson C, Inui M, Montagner M, Parenti AR, Poletti A, Daidone MG, Dupont S, Basso G, et al. The Hippo transducer TAZ confers cancer stem cell-related traits on breast cancer cells. Cell. 2011; 147:759-772.
19. Preca BT, Bajdak K, Mock K, Sundararajan V, Pfannstiel J, Maurer J, Wellner U, Hopt UT, Brummer T, Brabletz S, Brabletz T, Stemmler MP. A self-enforcing CD44s/ZEB1 feedback loop maintains EMT and stemness properties in cancer cells. Int J Cancer. 2015; 137:2566-2577.

20. Chanmee T, Ontong P, Itano N. Hyaluronan: A modulator of the tumor microenvironment. Cancer Lett. 2016; 375:20-30.

21. Hanahan D, Weinberg RA. Hallmarks of cancer: the next generation. Cell. 2011; 144:646-674.

22. Vigetti D, Karousou E, Viola M, Deleonibus S, De Luca G, Passi A. Hyaluronan: biosynthesis and signaling. Biochim Biophys Acta. 2014; 1840:2452-2459.

23. Heldin P, Basu K, Olofsson B, Porsch H, Kozlova I, Kahata K. Deregulation of hyaluronan synthesis, degradation and binding promotes breast cancer. J Biochem. 2013; 154:395-408.

24. Itano N, Sawai T, Miyaishi O, Kimata K. Relationship between hyaluronan production and metastatic potential of mouse mammary carcinoma cells. Cancer Res. 1999; 59:2499-2504.

25. Toole BP. Hyaluronan: from extracellular glue to pericellular cue. Nat Rev Cancer. 2004; 4:528-539.

26. Bourguignon LY. Hyaluronan-mediated CD44 activation of RhoGTPase signaling and cytoskeleton function promotes tumor progression. Semin Cancer Biol. 2008; 18:251-259.

27. Toole BP. Hyaluronan-CD44 Interactions in Cancer: Paradoxes and Possibilities. Clin Cancer Res. 2009; 15:7462-7468.

28. Weigel PH, Hascall VC, Tammi M. Hyaluronan synthases. J Biol Chem. 1997; 272:13997-14000.

29. Lien HC, Lee YH, Jeng YM, Lin CH, Lu YS, Yao YT. Differential expression of hyaluronan synthase 2 in breast carcinoma and its biological significance. Histopathology. 2014; 65:328-339.

30. Chanmee T, Ontong P, Mochizuki N, Kongtawelert P, Konno K, Itano N. Excessive hyaluronan production promotes acquisition of cancer stem cell signatures through the coordinated regulation of Twist and the transforming growth factor beta (TGF-beta)-Snail signaling axis. J Biol Chem. 2014; 289:26038-26056.

31. Zoltan-Jones A, Huang L, Ghatak S, Toole BP. Elevated hyaluronan production induces mesenchymal and transformed properties in epithelial cells. J Biol Chem. $2003 ; 278: 45801-45810$.

32. Kang Y, Siegel PM, Shu W, Drobnjak M, Kakonen SM, Cordon-Cardo C, Guise TA, Massague J. A multigenic program mediating breast cancer metastasis to bone. Cancer Cell. 2003; 3:537-549.

33. Kakizaki I, Kojima K, Takagaki K, Endo M, Kannagi R, Ito M, Maruo Y, Sato H, Yasuda T, Mita S, Kimata K, Itano N. A novel mechanism for the inhibition of hyaluronan biosynthesis by 4-methylumbelliferone. J Biol Chem. 2004; 279:33281-33289.

34. Urakawa H, Nishida Y, Wasa J, Arai E, Zhuo L, Kimata K, Kozawa E, Futamura N, Ishiguro N. Inhibition 
of hyaluronan synthesis in breast cancer cells by 4-methylumbelliferone suppresses tumorigenicity in vitro and metastatic lesions of bone in vivo. Int J Cancer. 2012; 130:454-466.

35. Barretina J, Caponigro G, Stransky N, Venkatesan K, Margolin AA, Kim S, Wilson CJ, Lehar J, Kryukov GV, Sonkin D, Reddy A, Liu M, Murray L, et al. The Cancer Cell Line Encyclopedia enables predictive modelling of anticancer drug sensitivity. Nature. 2012; 483:603-607.

36. Lee JK, Havaleshko DM, Cho H, Weinstein JN, Kaldjian EP, Karpovich J, Grimshaw A, Theodorescu D. A strategy for predicting the chemosensitivity of human cancers and its application to drug discovery. Proc Natl Acad Sci U S A. 2007; 104:13086-13091.

37. Clarke C, Madden SF, Doolan P, Aherne ST, Joyce H, O’Driscoll L, Gallagher WM, Hennessy BT, Moriarty M, Crown J, Kennedy S, Clynes M. Correlating transcriptional networks to breast cancer survival: a large-scale coexpression analysis. Carcinogenesis. 2013; 34:2300-2308.

38. Zhang G, He P, Tan H, Budhu A, Gaedcke J, Ghadimi BM, Ried T, Yfantis HG, Lee DH, Maitra A, Hanna N, Alexander HR, Hussain SP. Integration of metabolomics and transcriptomics revealed a fatty acid network exerting growth inhibitory effects in human pancreatic cancer. Clin Cancer Res. 2013; 19:4983-4993.

39. Sato M, Larsen JE, Lee W, Sun H, Shames DS, Dalvi MP, Ramirez RD, Tang H, DiMaio JM, Gao B, Xie Y, Wistuba, II, et al. Human lung epithelial cells progressed to malignancy through specific oncogenic manipulations. Mol Cancer Res. 2013; 11:638-650.

40. Kim ES, Kim MS, Moon A. TGF-beta-induced upregulation of MMP-2 and MMP-9 depends on p38 MAPK, but not ERK signaling in MCF10A human breast epithelial cells. Int J Oncol. 2004; 25:1375-1382.

41. Brown RL, Reinke LM, Damerow MS, Perez D, Chodosh LA, Yang J, Cheng C. CD44 splice isoform switching in human and mouse epithelium is essential for epithelial-mesenchymal transition and breast cancer progression. J Clin Invest. 2011; 121:1064-1074.

42. Spaderna S, Schmalhofer O, Wahlbuhl M, Dimmler A, Bauer K, Sultan A, Hlubek F, Jung A, Strand D, Eger A, Kirchner T, Behrens J, Brabletz T. The transcriptional repressor ZEB1 promotes metastasis and loss of cell polarity in cancer. Cancer Res. 2008; 68:537-544.

43. Okuda H, Kobayashi A, Xia B, Watabe M, Pai SK, Hirota S, Xing F, Liu W, Pandey PR, Fukuda K, Modur V, Ghosh A, Wilber A, et al. Hyaluronan synthase HAS2 promotes tumor progression in bone by stimulating the interaction of breast cancer stem-like cells with macrophages and stromal cells. Cancer Res. 2012; 72:537-547.
44. Hiraga T, Ito S, Nakamura H. Cancer stem-like cell marker CD44 promotes bone metastases by enhancing tumorigenicity, cell motility, and hyaluronan production. Cancer Res. 2013; 73:4112-4122.

45. Weilbaecher KN, Guise TA, McCauley LK. Cancer to bone: a fatal attraction. Nature Reviews Cancer. 2011; 11:411-425.

46. Ell B, Kang Y. SnapShot: Bone Metastasis. Cell. 2012; 151:690-690 e691.

47. Tarragona M, Pavlovic M, Arnal-Estape A, Urosevic J, Morales M, Guiu M, Planet E, Gonzalez-Suarez E, Gomis RR. Identification of NOG as a specific breast cancer bone metastasis-supporting gene. J Biol Chem. 2012; 287:21346-21355.

48. David CJ, Huang YH, Chen M, Su J, Zou Y, Bardeesy N, Iacobuzio-Donahue CA, Massague J. TGF-beta Tumor Suppression through a Lethal EMT. Cell. 2016; 164:1015-1030.

49. Porsch H, Bernert B, Mehic M, Theocharis AD, Heldin CH, Heldin P. Efficient TGFbeta-induced epithelialmesenchymal transition depends on hyaluronan synthase HAS2. Oncogene. 2013; 32:4355-4365.

50. Adamia S, Reiman T, Crainie M, Mant MJ, Belch AR, Pilarski LM. Intronic splicing of hyaluronan synthase 1 (HAS1): a biologically relevant indicator of poor outcome in multiple myeloma. Blood. 2005; 105:4836-4844.

51. Auvinen P, Rilla K, Tumelius R, Tammi M, Sironen R, Soini Y, Kosma VM, Mannermaa A, Viikari J, Tammi R. Hyaluronan synthases (HAS1-3) in stromal and malignant cells correlate with breast cancer grade and predict patient survival. Breast Cancer Res Treat. 2014; 143:277-286.

52. Koyama H, Hibi T, Isogai Z, Yoneda M, Fujimori M, Amano J, Kawakubo M, Kannagi R, Kimata K, Taniguchi S, Itano N. Hyperproduction of hyaluronan in neu-induced mammary tumor accelerates angiogenesis through stromal cell recruitment: possible involvement of versican/PG-M. Am J Pathol. 2007; 170:1086-1099.

53. Herr R, Wohrle FU, Danke C, Berens C, Brummer T. A novel MCF-10A line allowing conditional oncogene expression in 3D culture. Cell Commun Signal. 2011; 9:17.

54. Prat A, Parker JS, Karginova O, Fan C, Livasy C, Herschkowitz JI, He X, Perou CM. Phenotypic and molecular characterization of the claudin-low intrinsic subtype of breast cancer. Breast Cancer Res. 2010; 12:R68.

55. Wang Y, Klijn JG, Zhang Y, Sieuwerts AM, Look MP, Yang F, Talantov D, Timmermans M, Meijer-van Gelder ME, Yu J, Jatkoe T, Berns EM, Atkins D, et al. Gene-expression profiles to predict distant metastasis of lymph-node-negative primary breast cancer. Lancet. 2005; 365:671-679. 